\title{
Bluthochdruck
}

\section{Seitendifferenz signalisiert erhöhtes Mortalitätsrisiko}

— Ergibt sich bei der Blutdruckmessung an beiden Armen eine Seitendifferenz von 10 $\mathrm{mmHg}$, steigt das Mortalitätsrisiko um $24 \%$. Noch eine viel höhere prognostische Bedeutung haben Seitendifferenzen bei Patienten mit chronischen Nierenerkrankungen, belegen Ergebnisse einer von Prof. Rainer Kolloch, Bielefeld, vorgestellten Studie.

Im Rahmen der Untersuchung waren 421 internistische und nephrologische Patienten einer Klinikambulanz u. a. auf Seitendifferenzen untersucht und über sieben Jahre nachbeobachtet worden (Agarwal R et al. Hypertension 2008; 51: 657662). Dabei zeigte sich: Je ausgeprägter die Seitendifferenz, desto höher die Gesamtmortalität. Ein erhöhtes Risiko atherosklerotischer Gefäßveränderungen signalisieren auch ein erhöhter Pulsdruck und eine erhöhte Pulswellengeschwindigkeit (PWV), ihrerseits ein Indikator für versteifte Blutgefäße. In einer Langzeitstudie über 14 Jahre korrelierte eine erhöhte PWV deutlich mit kognitiven Leistungseinbußen. Möglicherweise zu Unrecht wird die PWVMessung derzeit vergleichsweise selten genutzt.

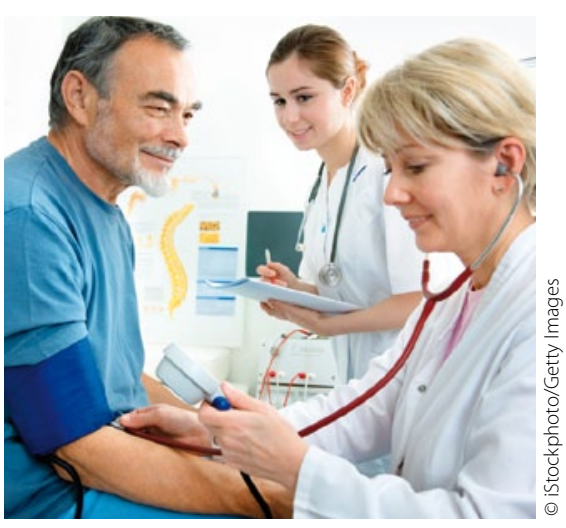

Bei einer Seitendifferenz von $10 \mathrm{mmHg}$ ist das Mortalitätsrisiko um $24 \%$ erhöht.

Im Kampf gegen atherosklerotische Gefäßläsionen und deren fatale Folgen zählt nach Darstellung Kollochs nicht allein die gute Blutdruckeinstellung, sondern auch die Art und Weise, wie diese erreicht wird. Ergebnisse der ACCOMPLISH-Studie wertet der Hochdruckspezialist als schlagkräftiges Argument für den Vorzug antihypertensiver Fixkombinationen. Zudem erwies sich in dieser Studie die Kombination von RAS-(Renin-Angiotensin-System-)Hemmer und Kalziumantagonist gegenüber der
Kombination RAS-Hemmer plus Diuretikum als überlegen.

\section{Günstige Effekte auf Plaquevolumen} Im Rahmen der OLIVUS-Studie ließ sich unter Olmesartan bei Patienten mit stabiler Angina pectoris und Hypertonie nach 14-monatiger Behandlung im Placebovergleich eine deutlich verzögerte Progression der Koronarsklerose nachweisen. Die Behandlung mit dem Sartan hatte größeren Einfluss auf das Plaquevolumen als die parallel durchgeführte Statinbehandlung.

Ungünstige Einflussfaktoren waren männliches Geschlecht, Alter > 65 und eine Diabetesdiagnose. Für eine Fixkombination von Olmesartan und Amlodipin (z. B. Vocado ${ }^{\circledR}$ ) sprechen auch von Kolloch vorgestellte Studiendaten, die unter dieser Kombination eine ausgeprägte Abnahme inflammatorischer Mediatoren wie CRP, diverse Interleukine oder VCAM-1 fanden.

- Ludger Riem

Quelle: Symposium „Gefäßprotektion -

Was kann eine moderne Hypertonietherapie leisten ?", Kongress der Deutschen Hochdruckliga, Köln, November 2011 (Veranstalter: Berlin-Chemie)
KHK-Patienten mit stabiler Angina pectoris \& Perkutane Koronarinterventionen (PCI) können bei KHK-Patienten mit stabiler Angina pectoris eine optimale Medikamentengabe ergänzen und drei Jahre lang den Anteil an Betroffenen mit Angina-pectoris-Anfällen reduzieren. Das berichtet eine Arbeitsgruppe des Deutschen Instituts für Medizinische Dokumentation und Information (DIMDI) in einem Health Technology Assessment (HTA). Allerdings hätten die Studien, die diesen Ergebnissen zugrundeliegen, nur eine eingeschränkte Aussagekraft. Experten empfehlen deshalb die PCI bei stabiler Angina pectoris nur eingeschränkt. Außerdem sei nicht zu erwarten, dass sich unter $\mathrm{PCl}$ die Rate von Todesfällen, Herzinfarkten oder
Schlaganfällen reduzierten. Goldstandard bleibt damit bei Angina pectoris ein akut wirksames Nitroglyzerin-Spray wie Nitrolingual ${ }^{\circledR}$, das auch in den europäischen und amerikanischen Versorgungsleitlinien empfohlen wird.

Pohl Boskamp

Phytotherapie bei Rhinosinusitis $>\mathrm{Ne}$ ben der Sekretolyse und der Wiederherstellung der mukoziliären Clearance ist die Eindämmung der überschießenden Entzündungsreaktion des Immunsystems ein wichtiges Therapieziel bei der Behandlung einer akuten oder chronischen Rhinosinusitis. Aktuelle Studiendaten belegen, dass das pflanzliche Arneimittel Sinupret ${ }^{\circledast}$ nicht nur sekretolytisch wirkt, sondern auch verschiedene Entzündungskaskaden bzw. Entzündungsmediatoren wie Zytokine hemmt. Ermöglicht wird dies durch die enthaltenen Bioflavonoide.

Bionorica SE

Metformin kontraindiziert? - Aufgrund von Unverträglichkeit oder Kontraindikationen wie Nierenerkrankung können ein Viertel aller Typ-2-Diabetiker kein Metformin verwenden. Galvus ${ }^{\circledast}$ (Vildagliptin) ist nun zur Monotherapie bei dieser Patientengruppe zugelassen. Die Entscheidung basiert auf der positiven CHMP-Beurteilung, die aufgrund klinischer Daten zu Wirksamkeit und Verträglichkeit bei über 3000 nicht vorbehandelten Patienten ausgesprochen wurde. Novartis 